\title{
Dynamic Analysis and Simulation of an Optically Levitated Rotating Ellipsoid Rotor in Liquid Medium
}

\author{
Qi ZHU ${ }^{1}$, Nan $\mathrm{LI}^{1}$, Heming $\mathrm{SU}^{1}$, Wenqiang $\mathrm{LI}^{1}$, and Huizhu $\mathrm{HU}^{1,2^{*}}$ \\ ${ }^{1}$ State Key Laboratory of Modern Optical Instrumentation, College of Optical Science and Engineering, Zhejiang \\ University, Hangzhou 310027, China \\ ${ }^{2}$ Quantum Sensing Center, Zhejiang Lab, Hangzhou 310000, China \\ *Corresponding author: Huizhu HU_ E-mail: huhuizhu2000@zju.edu.cn
}

\begin{abstract}
Optical trap, a circularly polarized laser beam can levitate and control the rotation of microspheres in liquid medium with high stiffness. Trapping force performs as confinement while the trapped particle can be analog to a liquid floated gyroscope with three degree-of-freedom. In this work, we analyzed the feasibility of applying optically levitated rotor in the system. We presented the dynamic analysis and simulation of an ellipsoid micron particle. The precession motion and nutation motion of a rotating ellipsoid probe particle in optical tweezers were performed. We also analyzed the attitude changes of an optically levitated ellipsoid when there was variation of the external torque caused by deviation of the incident light that was provided. Furthermore, the trail path of the rotational axis vertex and the stabilization process of a particle of different ellipticities were simulated. We compared the movement tendencies of particles of different shapes and analyzed the selection criteria of ellipsoid rotor. These analytical formulae and simulation results are applicable to the analysis of the rotational motion of particles in optical tweezers, especially to the future research of the gyroscope effect.
\end{abstract}

Keywords: Optical Tweezers; rotor; optically induced rotation; dynamic analysis

Citation: Qi ZHU, Nan LI, Heming SU, Wenqiang LI, and Huizhu HU, "Dynamic Analysis and Simulation of an Optically Levitated Rotating Ellipsoid Rotor in Liquid Medium,” Photonic Sensors, 2022, 12(2): 105-116.

\section{Introduction}

Rotational control has always attracted intense interest since the pioneering work achieved by Ashkin [1]. Spin angular momentum carried by circularly polarized light could lead to the constant rotation of particles in optical tweezers [2-7]. Due to low damping, microspheres can rotate at a rate exceeding $\mathrm{MHz}$, even $\mathrm{GHz}$ [8, 9]. Without mechanical contact, the balance of force and the torque acting on an optical levitated particle makes it a potent instrument with which to study environmental interactions [10-12]. Rotating particles in optical tweezers have been used for precise manipulation in a variety of applications, such as probes in microrheology [13-15], position alignment of a particular particle [16-18], and some laboratory experiments $[8,9,19]$.

Experiments of precession motion in optical tweezers have been observed [20-23]. The derivation of motional frequencies made it possible to divide precession angular velocity and nutation angular velocity from the signal while the trapped particle was confined by trapping light [22]. Such

Received: 22 March 2021 / Revised: 7 June 2021

(C) The Author(s) 2021. This article is published with open access at Springerlink.com

DOI: $10.1007 / \mathrm{s} 13320-021-0639-0$

Article type: Regular 
achievement made us wonder, whether the trapped particle could be analog to a liquid floated gyroscope rotor with 3-degree-freedom. A stable rotating particle in optical tweezers experiences optical torque and counterpart drag torque. The total amount of angular momentum transferred to a trapped particle will eventually equal the viscous torque. The quantization of torque has made it possible to calculate the features of certain particles and media. Theoretically, an optical levitated rotating particle resembles a suspended gyroscope.

There are many mature theories and research models to refer to in the dynamic analysis of spheres in optical tweezers [12, 24-26]; however, few are for ellipsoid particles. A sphere can rotate along any random axis, whereas an ellipsoid particle has a fixed axis of rotation and a corresponding precession angular velocity, self-rotation velocity, and nutation angle, for the latter experiencing no spherical symmetry and more suitable for rotor. An isotropy ellipsoidal particle with uniform mass distribution has its centroid coincide with its geometric center. It has been observed and analyzed in detail that optical force may cause orbital rotation, but few research has been made on the rotating and precession motion of trapped particle in optical tweezers.

Previous experiments have shown that a levitated rotating particle's rotational axis is not strictly fixed in the inertial space, partially due to its precession motion and nutation motion [22, 23, 27]. A levitated micro-sized sphere can measure the external torque by detecting the variation of the rotation rate and precession angle. Compared with a traditional rotor, a laser-levitated particle experiences only rotational drag torque and no effect is brought about by the machinery's friction, which can dramatically increase its accuracy. Furthermore, in a pressure-controllable environment, we can reduce the influence of the flow disturbance by controlling the temperature and reducing the pressure. However, the dynamic analysis and simulation of a high-speed rotating sphere in a high vacuum have not been studied.

In this work, we presented the levitated micronsized rotor equation of motion in air. We chose an ellipsoidal particle as the analysis object. The analysis and simulation were carried out to demonstrate the dynamic process of the trapped rotating particle while the external torque was applied.

\section{Levitated micron-sized rotor equation of rotation motion}

To describe the orientation of a rigid body, i.e., a levitated particle, we should create a coordinate system attached to the rotating body. Taking coordinate system $x_{1} y_{1} z_{1}$ as the origin coordinate system, we could create the inertial coordinate system using $z-y^{\prime \prime}-z^{\prime}$ convention. The rotational motion of the particle can be described by three parameters: (1) the spin angular velocity $\varphi$ of the rotating particle, $(2)$ the precession angular velocity $\dot{\psi}$ of the rotational axis of the rotating sphere, and (3) the nutation angle $\theta$ between the spin and precession axes.

The Euler mechanics equation of a rotating rotor in the inertial space is as follows:

$$
\left\{\begin{array}{l}
I_{1}(\ddot{\theta}-\dot{\psi} \cos \theta \sin \theta)+I_{3} \dot{\psi} \sin \theta(\dot{\varphi}+\dot{\psi} \cos \theta)=\tau_{\mathrm{re}_{1}} \\
I_{1}(\ddot{\psi} \sin \theta+2 \dot{\psi} \dot{\theta} \cos \theta)-I_{3} \dot{\theta}(\dot{\varphi}+\dot{\psi} \cos \theta)=\tau_{\mathrm{re}_{2}} \\
I_{3} \frac{\mathrm{d}}{\mathrm{d} t}(\dot{\varphi}+\dot{\psi} \cos \theta)=\tau_{\mathrm{re}_{3}}
\end{array}\right.
$$

where $I_{1}$ and $I_{3}$ represent the momentums of

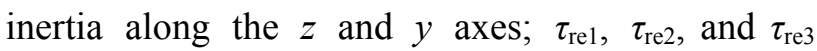
represent the moment components in the inertial coordinate system. An ideal rotating rotor experiences resultant torque $\tau_{\mathrm{re}}=0$, so $\tau_{\mathrm{re}_{1}}=\tau_{\mathrm{re}_{2}}=\tau_{\mathrm{re}_{3}}=0$. If $\theta=\theta_{0}$ is set, a particular solution can be obtained as follows:

$$
\begin{gathered}
\dot{\psi}=\dot{\psi}_{0} \\
\dot{\varphi}=\dot{\varphi}_{0}=-\frac{I_{3}-I_{1}}{I_{1}} \dot{\psi}_{0} \cos \theta .
\end{gathered}
$$


An ideal rotating particle will spin at a constant rate while its spin axis rotates around the precession axis, i.e., the optical axis at a constant rate. When $I_{1}=I_{3}$, a particle can be described as an absolute sphere. Such a particle will only have precession angular velocity, while no spin angular velocity has evolved. Only an ellipsoidal particle with $I_{1} \neq I_{3}$ will be included in the following discussion.

When a circularly polarized laser beam captures a birefringent particle with an initial angular velocity and a rotating axis or the circularly polarized laser beam deflects at a certain angle, the variation of the resultant torque on the particle will lead to the variation of the motion state. Elaborating such a process requires a third coordinate system to express the kinematic relationship among the incident laser beam, the particle's angular momentum, and the particle's motion.

Here, three coordinate systems that originate at centroid $\mathrm{O}$ are introduced, as shown in Fig. 1: (1) coordinate system $x_{1} y_{1} z_{1}$, which represents a stationary coordinate system in which the $z_{1}$ axis always coincides with the optical axis, and the other two axes point to fixed orientations; (2) coordinate system $x_{2} y_{2} z_{2}$, which represents an angular momentum coordinate system in which the $z_{2}$ axis always coincides with the angular momentum vector of the particle; (3) coordinate system $x_{3} y_{3} z_{3}$, which represents a coordinate system that is rigidly connected to particles. Here, $\rho$ represents the angle between the moment of the momentum axis and the presumed optical axis, and $\kappa$ represents the transverse angle between the optical angle and the rotational coordinate system, where $\dot{\kappa}$ represents the relative rotational speed between the rotational coordinate and static rotational coordinate systems. The Euler mechanics equation of the angular momentum coordinate system $x_{2} y_{2} z_{2}$ is as follows:

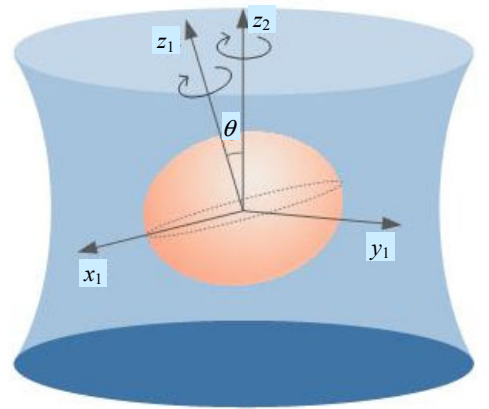

(a)

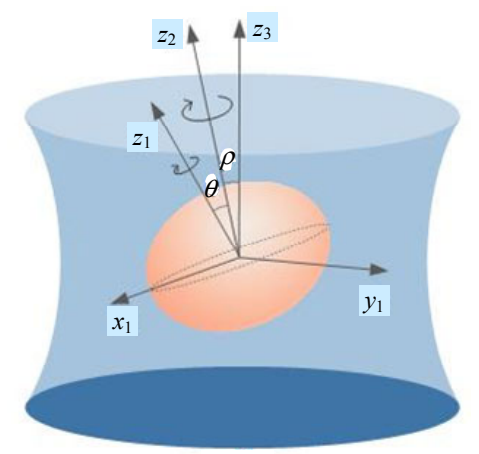

(b)

Fig. 1 A steady rotating particle, with steady rotation and precession. Here, in the coordinate system $x_{2} y_{2} z_{2}$ and $x_{1} y_{1} z_{1}$, axes $x_{1}, y_{1}$, and axis $z_{2}$ are marked, whereas axes $x_{2}$ and $y_{2}$ are omitted (a) and the transition of the coordinate system of a rotating particle when the laser beam deviates by angle $\rho$

(b). Here, in the coordinate systems $x_{1} y_{1} z_{1}, x_{2} y_{2} z_{2}$, and $x_{3} y_{3} z_{3}$, axes $x_{1}, y_{1}, z_{1}, z_{2}$ and axis $z_{3}$ are marked, whereas axes $x_{2}, y_{2}, x_{3}$, and $y_{3}$, are omitted.

$$
\left[\begin{array}{c}
0 \\
0 \\
\dot{H}
\end{array}\right]+\left[\begin{array}{ccc}
0 & -\dot{\kappa} \cos \rho & \dot{\rho} \\
\dot{\kappa} \cos \rho & 0 & \dot{\kappa} \sin \rho \\
-\dot{\rho} & -\dot{\kappa} \sin \rho & 0
\end{array}\right]\left[\begin{array}{l}
0 \\
0 \\
H
\end{array}\right]=\left[\begin{array}{c}
\tau_{\mathrm{re}_{x_{2}}} \\
\tau_{\mathrm{re}_{y_{2}}} \\
\tau_{\mathrm{re}_{z_{2}}}
\end{array}\right]
$$

where $\tau_{\mathrm{re}_{x_{2}}}, \tau_{\mathrm{re}_{y_{2}}}$, and $\tau_{\mathrm{re}_{z_{2}}}$ are the projections of the resultant torque $\tau_{\mathrm{re}}$ on the angular momentum coordinate system, and $H$ is the moment of momentum. Since $\tau_{\mathrm{re}_{x_{2}}}, \tau_{\mathrm{re}_{y_{2}}}$, and $\tau_{\mathrm{re}_{z_{2}}}$ are functions of $\dot{\varphi}, \dot{\psi}$, and $\theta$, the kinematic relation of the Euler angle $(\varphi, \psi, \theta)$ is essential to obtain a closed-form solution. The equations of equilibrium and the kinematic relations among the torque, angular momentum, and Euler angles are as follows: 


$$
\begin{aligned}
& \dot{\rho}=\frac{1}{H} \tau_{\mathrm{re}_{x_{2}}} \\
& \dot{\kappa}=\frac{1}{H \sin \rho} \tau_{\mathrm{re}_{y_{2}}} \\
& \dot{H}=\tau_{\mathrm{re}_{z_{2}}} \\
& \dot{\psi}=\frac{H}{I_{1}}-\frac{\cot \theta}{H}\left(\tau_{\mathrm{re}_{y_{2}}} \sin \psi+\tau_{\mathrm{re}_{x_{2}}} \cos \psi\right)-\tau_{\mathrm{re}_{y_{2}}} \frac{\cot \rho}{H} \\
& \dot{\theta}=\frac{1}{H}\left(\tau_{\mathrm{re}_{y_{2}}} \cos \psi-\tau_{\mathrm{re}_{x_{2}}} \sin \psi\right) \\
& \dot{\varphi}=\frac{H\left(I_{1}-I_{3}\right)}{I_{1} I_{3}} \cos \theta+\frac{1}{H \sin \theta}\left(\tau_{\mathrm{re}_{y_{2}}} \sin \psi+\tau_{\mathrm{re}_{x_{2}}} \cos \psi\right) .
\end{aligned}
$$

When no external torque is applied, the particle would be in an ideal rotation state. From (5), we can have the following equations:

$$
\begin{aligned}
& \rho=\rho_{0} \\
& \kappa=\kappa_{0} \\
& H=H_{0} \\
& \dot{\psi}=\frac{H}{I_{1}}=\dot{\psi}_{0} \\
& \theta=\theta_{0} \\
& \dot{\varphi}=-\frac{I_{3}-I_{1}}{I_{3}} \dot{\psi}_{0} \cos \theta_{0}
\end{aligned}
$$

where $\rho, H, \kappa$, and $\theta$ are the slow variables, whereas $\psi$ and $\varphi$ are the fast variables whose harmonic functions have zero mean value. Obtaining the analytical solution of each variable is difficult. Here, the averaging method can be used to eliminate the time-varying high-frequency component in the external torque to obtain the analytical solution or discuss motion characteristics.

\section{Rotational motion of a particle when only optical torque is applied}

When a particle receives only optical torque, $\tau_{\mathrm{re}_{x_{2}}}, \tau_{\mathrm{re}_{y_{2}}}$, and $\tau_{\mathrm{re}_{z_{2}}}$ can be determined as follows:

$$
\left\{\begin{array}{l}
\tau_{\mathrm{re}_{x_{2}}}=-M \sin \rho \\
\tau_{\mathrm{re}_{y_{2}}}=0 \\
\tau_{\mathrm{re}_{z_{2}}}=M \cos \rho
\end{array}\right.
$$

where $M$ represents the external torque in the static coordinate system. When a particle is trapped in optical traps, it arrives with an instantaneous angular speed that can determine the initial values of $\dot{\varphi}, \dot{\psi}$, and $\theta$, and an instantaneous rotational axis that can determine the initial value of $\rho$ and $\kappa$. Placing (6) in (5):

$$
\begin{aligned}
& \dot{\rho}=\frac{M}{H} \sin \rho \\
& \dot{\kappa}=0 \\
& \dot{H}=M \cos \rho \\
& \dot{\psi}=\frac{H}{I_{1}}-\frac{M}{H} \cot \theta \sin \rho \cos \psi \\
& \dot{\theta}=-\frac{M}{H} \sin \rho \sin \psi \\
& \dot{\varphi}=\frac{H\left(I_{1}-I_{3}\right)}{I_{1} I_{3}} \cos \theta+\frac{M \sin \rho}{H \sin \theta} \cos \psi .
\end{aligned}
$$

It has been well explained that $\rho$ will tend to 0 , meaning the optical torque performs a role of centering. Hence, in a high vacuum, whatever initial state the trapped particle is in, its rotational axis will finally coincide with the optical axis. However, when only the optical torque is considered, the particle will not reach a steady rotational state. Theoretically, the rotational velocity increases indefinitely; however, in practice, this proves impossible.

\section{Total torque exerted on trapped particle}

Tangential force exerted on the trapped particle will cause orbital rotation in optical trap, but the Magus effect and rotor effect are decoupling for isotropy ellipsoidal particle with uniform mass distribution. To reduce the effect of particle's translational motion as far as possible, we set $T=$ $300 \mathrm{~K}$ [5]. We use an ellipsoidal $\mathrm{SiO}_{2}$ particle, with a major axis of $5 \mu \mathrm{m}$ and a minor axis of $1 \mu \mathrm{m}$ to $4 \mu \mathrm{m}$, as the simulation object.

However, a rotating particle trapped in optical tweezers experiences a moment of external torque 
that consists of resistance torque $\tau_{\text {drag }}$ and optical torque $\tau_{\mathrm{opt}}$ brought about by a circular polarized laser beam. The Gaussian varying random noise due to Brownian motion can be negligible compared with the two components above. The trapped particle will reach a steady state when the optical torque and rotational Stokes drag torque are balanced.

The optical torque is induced by the change of the laser beam's photon polarization state $\sigma$ while traversing a birefringent particle: $\tau_{\mathrm{opt}}=\sigma P / \omega$, where $P$ is the total optical power at the particle, and $\omega$ is the angular frequency of the light $[28,29]$.

The rotational drag torque of a particle rotating in a fluid at a low Reynolds number is $\tau_{\text {drag }}=8 \pi \eta a^{3} \Omega$, where $\eta$ is the kinematic viscosity, $a$ is the radius of the particle, and $\Omega$ is the angular speed [29]. Therefore, the external torque's projection on the angular momentum coordinate system can be expressed as follows:

$$
\begin{aligned}
& \tau_{\mathrm{re}_{2}}=\tau_{\mathrm{opt}_{2}}-\tau_{\mathrm{drag}_{2}} \\
& =\left[\begin{array}{c}
\tau_{\mathrm{opt}}(-\sin \rho) \\
0 \\
\tau_{\mathrm{opt}} \cos \rho
\end{array}\right]-8 \pi \eta a^{3}\left[\begin{array}{c}
\dot{\theta} \cos \psi+\dot{\varphi} \sin \theta \sin \psi \\
-\dot{\varphi} \sin \theta \cos \psi+\dot{\theta} \sin \psi \\
\dot{\psi}+\dot{\varphi} \cos \theta
\end{array}\right] \\
& =\left[\begin{array}{c}
\tau_{\mathrm{opt}}(-\sin \rho)-8 \pi \eta a^{3}(\dot{\theta} \cos \psi+\dot{\varphi} \sin \theta \sin \psi) \\
8 \pi \eta a^{3}(\dot{\varphi} \sin \theta \cos \psi+\dot{\theta} \sin \psi) \\
\tau_{\mathrm{opt}} \cos \rho-8 \pi \eta a^{3}(\dot{\psi}+\dot{\varphi} \cos \theta)
\end{array}\right] .
\end{aligned}
$$

where $\tau_{\mathrm{opt}_{2}}$ represents the optical torque in inerial coordinate system, and $\tau_{\mathrm{drag}_{2}}$ represents the drag torque in the inerial coordinate system. We use the averaging method to eliminate the time-varying high-frequency component in $\rho$ and $\kappa$, and the small-angle approximation is as follows:

$$
\begin{gathered}
\dot{\rho}=\frac{\tau_{\text {opt }}(-\sin \rho)}{H} \\
\dot{\kappa}=0 .
\end{gathered}
$$

Solving the equation, it can be deduced that the deviation angle $\rho$ and the nutrition angle $\theta$ will tend to 0 . This shows that a rotating particle in optical tweezers has characteristics of precession and inertia.

\section{Orientation process of the particle}

When an ellipsoidal particle is trapped, the major axis is unnecessarily coincided with the optical axis. To determine the stable state of the trapped particle, the instantaneous motion state variables should be analyzed.

The approximation solution of $\rho$ shows that the damping time depends only on the magnitude of the optical force and the angular momentum of the trapped particle. By substituting (9) to (7), the approximation of $\theta$ under given external conditions can, hereby, be obtained:

$$
\dot{\theta}=\frac{8 \pi \eta a^{3}}{H} \dot{\varphi} \sin \theta .
$$

The phase path of the trapped ellipsoidal particle in the $\theta-\dot{\theta}$ plane shows that the damping process after trapping can be treated as the stabilization of the particle. The variation of the nutation angle is determined by the sign of the self-angular velocity of the particle, which is determined by the ratio of $I_{1}$ and $I_{3}$.

When $I_{1} / I_{3}>1$, the minor inertial principal axis of the particle is treated as the axis of rotation. The particle's spin angular velocity and precession angular velocity have the same sign. Figure 2 shows that the nutation angle $\theta$ will reach the stabilizing value of $\pi / 2$ over time. When the nutation angle reaches $\pi / 2$, the particle reaches an unstable equilibrium position that can only be sustained when $\dot{\theta}=0$. Such an equilibrium state will be vandalized by any small disturbance; thus, a "vertical" ellipsoidal particle will gradually lie down and rotate around its major inertial principal axis. When we set the initial nutation angle $\theta_{0}=\pi / 2$, the variation of this depended on the initial value of the spin angular velocity and the precession angular velocity.

Figures 2(b) and 2(c) show that when $I_{1} / I_{3}<1$, which indicates that the maximum inertial principal axis of the particle is the polar axis of rotation, the 
particle experiences counter-precession, in which its self-angular velocity and precession angular velocity have different signs. The polar axis will coincide with $\mathrm{OZ}_{2}$ of the angular momentum coordinate

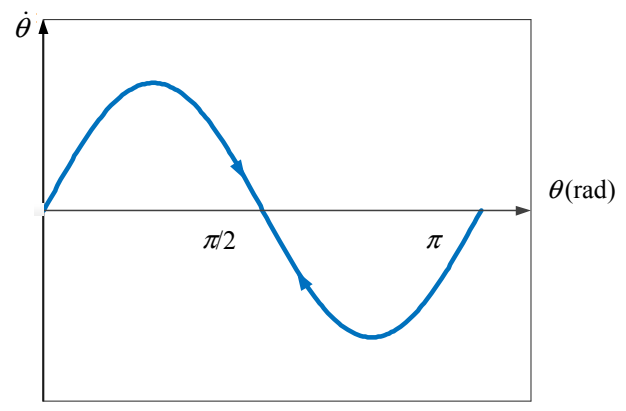

(a)

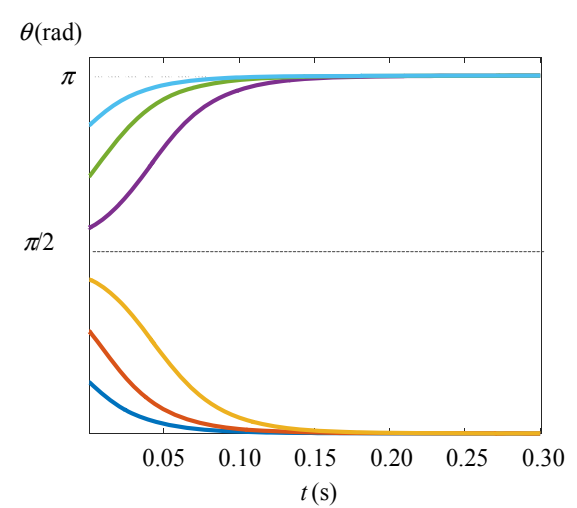

(c) system, whereas $O Z_{2}$ will gradually coincide with the optical axis $O Z_{1}$ as $\rho$ gradually decreases to zero. The damping process achieves stabilization of the particle.

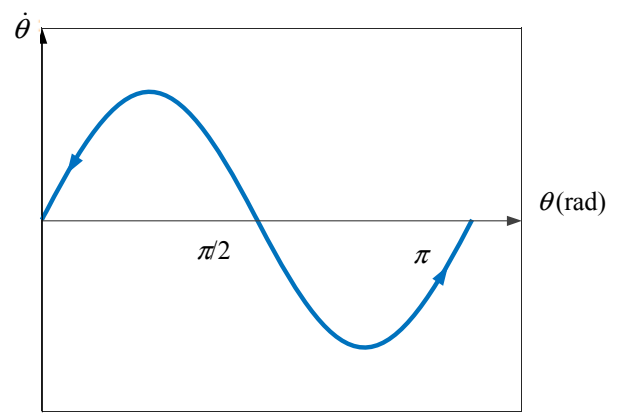

(b)

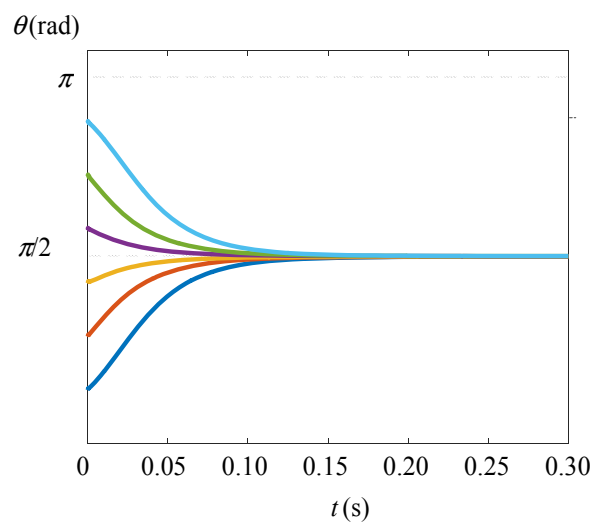

(d)

Fig. 2 Phase path and nutation angle of particles of different shapes: (a) the phase path in the $\theta-\dot{\theta}$ plane when $I_{1} / I_{3}>1$, (b) the phase path in the $\theta-\dot{\theta}$ plane when $I_{1} / I_{3}<1$, (c) the change of the nutation angle $\theta$ with time when $I_{1} / I_{3}<1$, and (d) the change of the nutation angle $\theta$ with time when $I_{1} / I_{3}>1$.

The most obvious problem lies in the fact that the pole of the captured particle cannot be fixed in advance. Suppose a particle has the north and south poles on its vertex. When the particle is captured by a laser beam, the initial orientation of the pole at a particular moment cannot be preset. Although the particle will finally reach equilibrium, determining which pole will be upward after stabilization is impossible. When $I_{1} / I_{3}>1$, an ellipsoidal particle has two major axes and one minor axis. Figures 2(a) and 2(d) show that whatever the initial nutation angle is, the particle tends to lie down to the $X O Y$ plane. It is impossible to fix the polar axis. Figure 2(d) shows that when $\theta_{0}<\pi / 2$ or $\theta_{0}>\pi / 2$, particles of different initial nutation angles will have its pole of the minor axis tend to the optical axis along the shortest path within $0.15 \mathrm{~s}$. Although it proves the good centering effect for particle of $I_{1} / I_{3}>1$, Fig. 3 shows that the polar orientation is determined by the initial orientation.

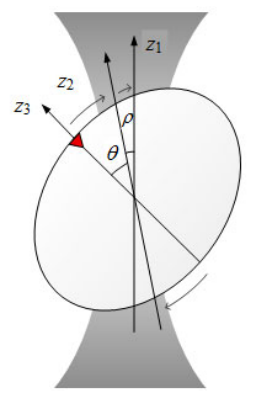

(a)

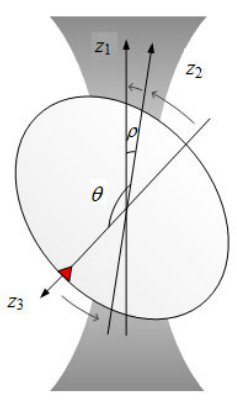

(b)
Fig. 3 Moving process of $\theta$ when a different initial position is taken. 
The damping force acts as a centering system. Particles of different initial rotational motions under the same condition will eventually reach the same equilibrium: $\rho$ tends to 0 and the nutation angles reach the same value. The time of the stabilization process depends on the ratio of $I_{1}$ and $I_{3}$.

\section{Stabilization process of the "Horizontal" particle}

A particle's ellipticity determines the ratio of $I_{1}$ and $I_{3}$, which will certainly affect the stabilization process. We will assume that the particle always rotates along one of its axes of symmetry when trapped. The two other cases of a particle with $I_{1} / I_{3}>1$ and a particle of $I_{1} / I_{3}<1$ will be discussed separately.

The particle of $I_{1} / I_{3}<1$ rotates along the maximum inertial principal axis, i.e., a micro-disk or flattened ellipsoid. We have discussed that a particle with such characteristics experienced its polar axis gradually leaning to the optical axis. In Fig. 4, we set the particle's major axis at $10 \mu \mathrm{m}$, its minor axis at $2 \mu \mathrm{m}, 4 \mu \mathrm{m}, 6 \mu \mathrm{m}$, and $8 \mu \mathrm{m}$, its initial spin angular velocity at $20 \mathrm{rad} / \mathrm{s}$, its initial precession angular velocity at $1000 \mathrm{rad} / \mathrm{s}$, and its initial nutation angle at $1 \mathrm{rad}$.

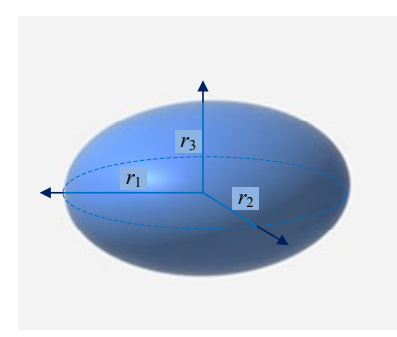

(a)

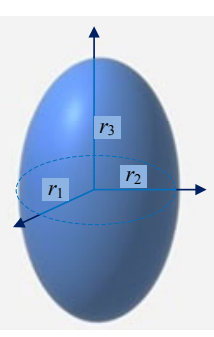

(b)
Fig. 4 Illustration of particles of two kinds: (a) illustration of "horizontal particle", which has $r_{1}=r_{2}>r_{3}$ and (b) illustration of "vertical particle", which has $r_{1}=r_{2}<r_{3}$.

According to the dynamic simulation, a particle of a different shape with the same initial state will eventually reach an equilibrium state in which its polar axis will almost coincide with the optical axis. We compared the centering process of particles with different shapes. After a certain period of stabilization $(0.05 \mathrm{~s}$ to $0.15 \mathrm{~s})$, the particle finally rotated at a fixed spin angular velocity and a precession angular velocity. The nutation angle of each equilibrium state would appear slightly different.

An ellipsoidal particle with a major axis of $10 \mu \mathrm{m}$ and a minor axis of $8 \mu \mathrm{m}$ can be treated as a sphere, whereas an ellipsoidal particle with major axis of $10 \mu \mathrm{m}$ and a minor axis of $2 \mu \mathrm{m}$ can be approximated to a disk. Figure 5 shows the initial state and equilibrium state of ellipsoids with the major axis of $10 \mu \mathrm{m}$ and minor axis of $8 \mu \mathrm{m}, 6 \mu \mathrm{m}$, $4 \mu \mathrm{m}$, and $2 \mu \mathrm{m}$. Though it seems like ellipsoid of different minor axes reaches the similar equilibrium state in Fig. 5, the density of the trail path shown in the right side shows that it takes a particle with the greater minor axis longer time than a particle with the smaller minor axis to reach a steady state. To further understand the dynamic process, we compared the delay time of particles of each shape. From the simulation of the trace of the vertex, as shown in Fig. 6, the greater the difference between $I_{1}$ and $I_{3}$ is, the more scattered the trace is.

Figure 6 can also show that the nutation angle in an equilibrium state is not exactly zero; it is also not the same for particles of different shapes. Although the difference is relatively subtle, as shown in Fig. 5(b), the nutation angle at the equilibrium state decreases with a decrease in particle's short axis.

Therefore, it can be concluded that a particle of a smaller $I_{1} / I_{3}$ can reach a steady rotational state faster than a particle of $I_{1} / I_{3}$ approaching 1 and is less sensitive to the angle variation. 

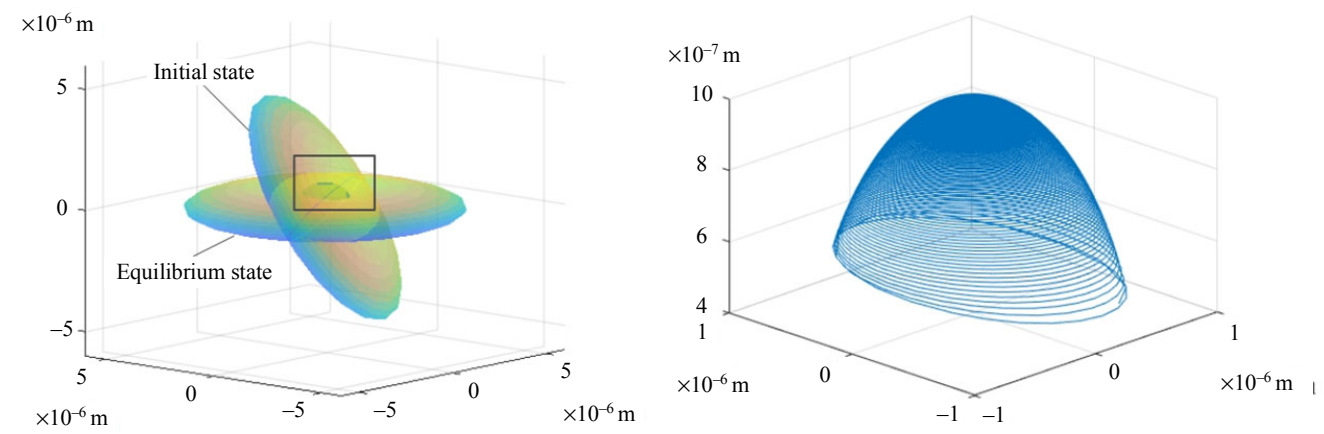

(a)
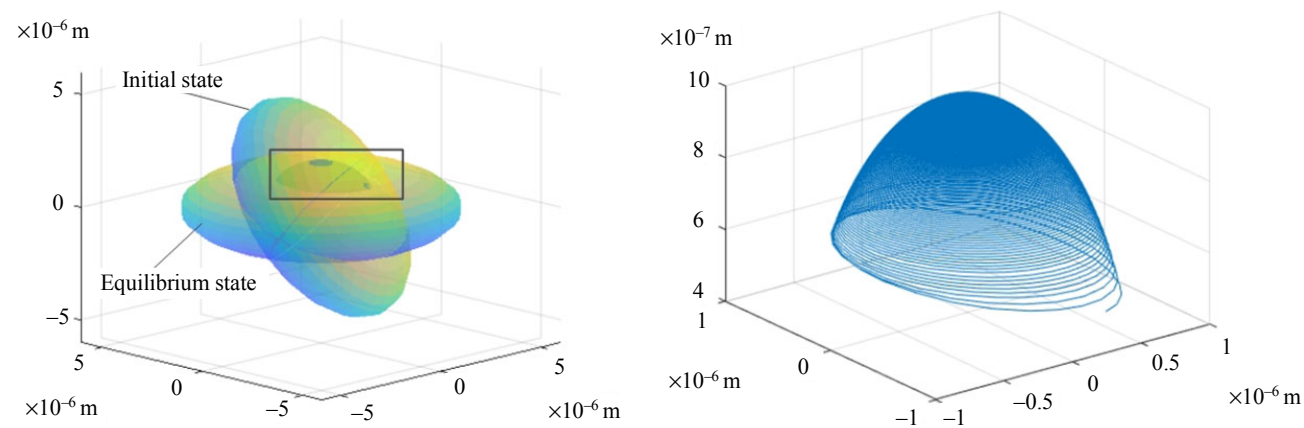

(b)
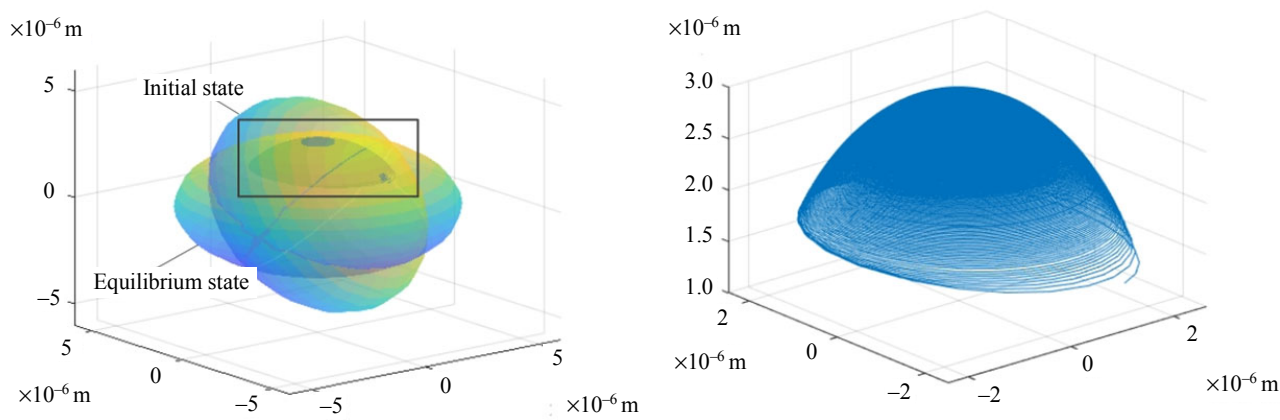

(c)
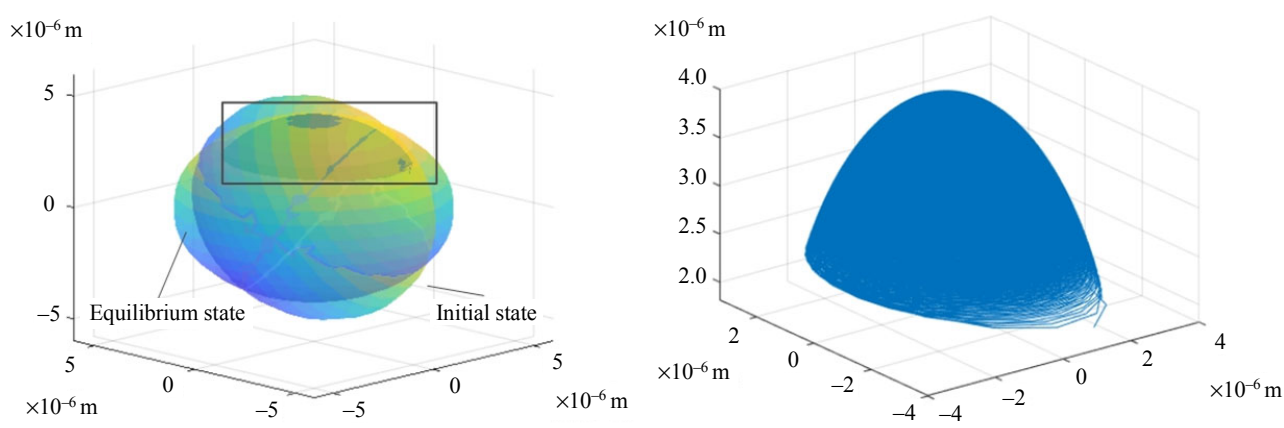

(d)

Fig. 5 Initial and equilibrium states and the motion path of the vertex of a particle with different minor axes: (a) the initial and equilibrium states and the motion path of the vertex of a particle with a minor axis of $2 \mu \mathrm{m}$, (b) the initial and equilibrium states and the motion path of the vertex of a particle with a minor axis of $4 \mu \mathrm{m}$, (c) the initial and equilibrium states and the motion path of the vertex of a particle with a minor axis of $6 \mu \mathrm{m}$, and (d) the initial and equilibrium states and the motion path of the vertex of a particle with a minor axis of $8 \mu \mathrm{m}$. 


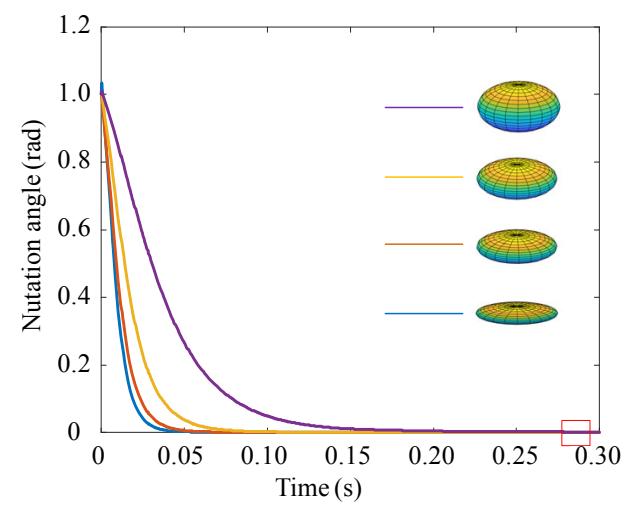

(a)

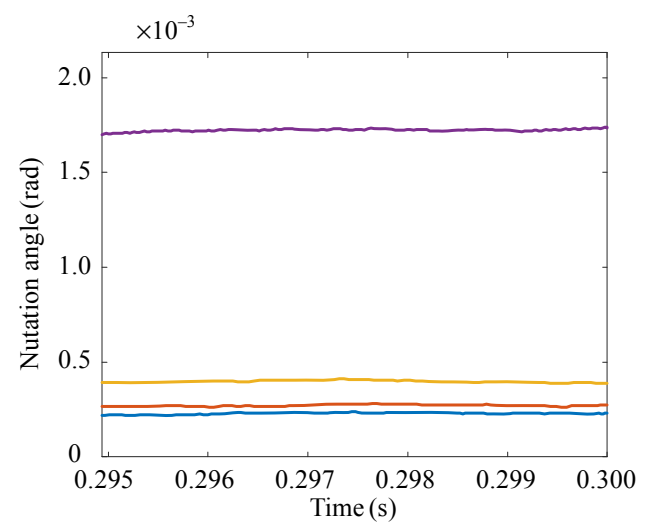

(b)

Fig. 6 Nutation angle of different shapes: (a) the variation of the nutation angle of particles of different shapes and (b) the enlarged image of the red box in (a).

\section{Stabilization process of a "vertical" particle"}

A vertical particle indicates that a particle is rotating along its minimum inertial axis, i.e., micro-rod or prolate ellipsoid. Mathematically, the approximation shows that a particle of $I_{1} / I_{3}>1$ will finally have its nutation angle tending toward $\pi / 2$. However, in the previous experiments, there were cases of a prolate ellipsoid rotating along its minimum inertial axis when trapped in a laser beam. Such a conflict should be resolved before further research.

The phase path in the $\theta-\dot{\theta}$ plane and the approximation of $\rho$ show that the trail of a particle's pole is much determined by the initial values of $\theta$ and $\rho$. A simulation calculation aiming at different initial values of $\theta$ and $\rho$ is carried out, as shown in Fig. 7. The initial values of the deviation angle $\rho$ and the nutation angle $\theta$ of the trapping light are initially set in a range of $0-$ $\pi / 2$. The simulation result of an ellipsoid particle with a major axis of $5 \mu \mathrm{m}$ and two minor axes of $4 \mu \mathrm{m}$ also shows that, when the nutation angle $\theta$ reaches $\pi / 2$, no self-rotation, but only precession motion exists, indicating that the particle will find its way to reach a state that rotates along its major inertial axis and maintain steady rotation.

Multiple simulation results show that it takes longer for $\theta$ having an initial value closer to $\pi / 2$ to achieve stable rotation (in Fig. 7). The two trail paths on right visually show that a larger initial nutation angle requires longer time for centering. Therefore, we expected that a rather smaller initial value of the nutation angle might help a vertical particle remain at its initial trapping attitude and rotate without leaning horizontally.

After multiple simulation comparisons and comprehensive analysis, our conjecture was verified. A particle with small initial nutation and deviation angles would not lean down when trapped but had its nutation angle slightly reduced to 0 and had a steady precession angular velocity and a rather small self-rotational angular velocity that corresponded to the steady rotational state of a "horizontal" particle. We also prolonged the simulation time and found out that such a state would be retained.

The deviation angle $\rho$ plays a crucial role in simulation. Such a steady rotational state occurs only when the initial deviation angle is sufficiently small. Simulation shows that the minimum precondition requires $\rho$ being $\sim 10^{-3} \mathrm{rad}$ under our simulation condition to have particles of the initial nutation angle $\theta$ of $\sim 10^{-4}$ rad and retain steady rotation, as shown in Fig. 8. 


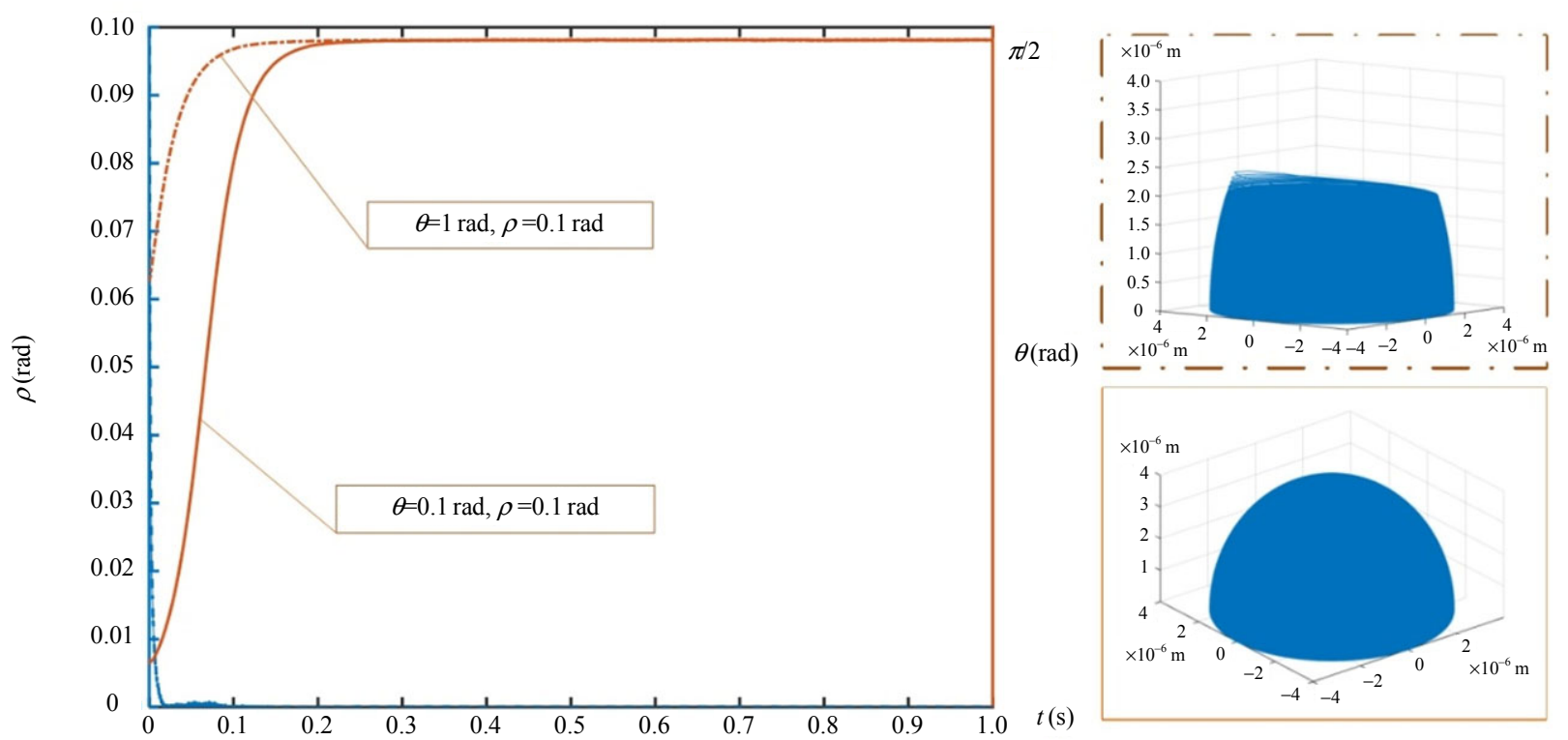

Fig. 7 Changes of $\theta$ and $\rho$ under different initial values and the simulation of the moving trail of a particle's pole.

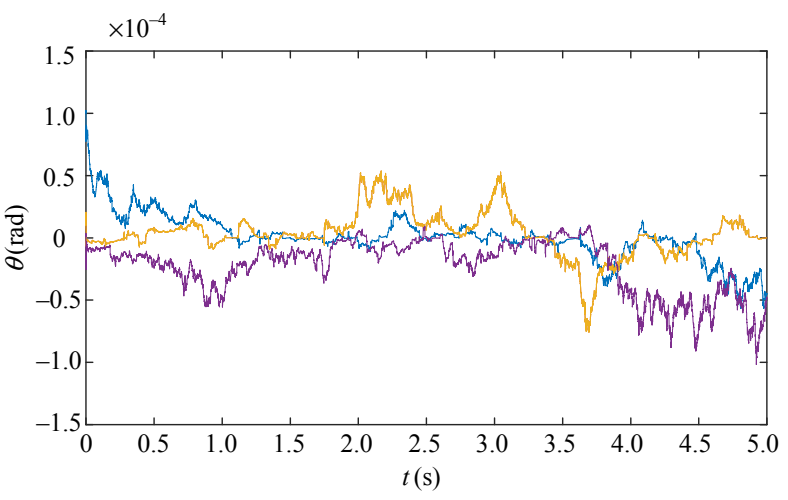

Fig. 8 Changes of $\theta$. Purple, yellow, and blue lines represent the changes of the nutation angle when initial values of $0.01,0.001$, and $0.0001 \mathrm{rad}$ are taken, respectively $(\rho=$ $0.001 \mathrm{rad})$.

\section{Rotation analysis}

In our simulation, we chose a $\mathrm{SiO}_{2}$ ellipsoidal particle to be the simulation object. We divided micron size rotor into two categories: horizontal particle and vertical particle, as shown in Fig. 4. From theory and simulation, horizontal particle's procession axis will stabilize after oscillating for a period and reach an equilibrium state under the common effect of external torque and the torque of resistance. An ellipsoid particle will achieve a fixed precession angular velocity, a self-rotational angular velocity, and a nutation angle after a short period of time. Mathematically, the final state has little to do with the deviation angle of the polarized light but rather is affected by the damping force, shape of the particle, and initial value of the nutation angle when the particle is trapped.

We have divided ellipsoid particles into vertical particles and horizontal particles and simulated the rotational process of two such kinds of particles, respectively. A horizontal particle rotates along its major inertial axis when trapped, whereas a vertical particle rotates along its minor inertial axis. A horizontal particle's rotational axis will eventually coincide with the optical axis when the trapping beam deflects. Such a characteristic makes a horizontal particle more likely to center than a vertical particle.

One of the obvious problems for the centering process of the horizontal particle is that if a particle, like a gyro rotor, has a fixed pole in advance, the orientation of the pole after centering can only be determined by the attitude of the particle before being trapped. We have yet to find corresponding countermeasures. This might become a future focus of the gyroscope effect research in optical tweezers.

We compared the dynamic of different 
ellipsoidal horizontal particles of different shapes in optical tweezers. An ellipsoidal particle with greater short axis rotates more stable and less sensitive than that with an ellipsoidal particle of smaller short axis, indicating that choosing a particle that is almost a sphere, but in which $I_{1}$ is slightly smaller than $I_{3}$, to study the gyroscopic effect of a laser levitated particle is necessary. An ellipsoidal particle with greater short axis needs more time to center than an ellipsoidal particle with smaller short axis, and it leaves a larger nutation angle, indicating that this type of shape is suitable for the further research.

Our calculation indicated that a vertical ellipsoidal particle would lie down to the $X O Y$ plane, making it an appropriate subject for the further simulation and calibration of the gyroscope effect research; this might seem to contradict some of the laboratory results. We carried out many simulations and found out that a vertical ellipsoidal particle might remain vertical and rotate when the initial value of the nutation angle was small enough for a certain deviated light. Other factors might be involved in the practical situation, but, nevertheless, such a particular case exists.

\section{Conclusions}

Analytical theory of a rotating sphere in optical tweezers has been subject to numerical calculations, while the same is not true for an ellipsoid particle. In this work, we derived the equations of rotation motion of a non-sphere. Formula deduction and simulation of movement of an ellipsoid particle in optical tweezers, when external torque and drag torque were applied, are presented. We introduced a third coordinate to further elaborate the motion of a rotating particle in optical tweezers.

While calculation simulation shows that the drag force acts as a centering system that can pull back the polar axis of the particle when the beam is deflected, simulation shows the trail path of the ellipsoid probe particle in liquid. A rotating particle in optical tweezers can be analogous to a relatively high-speed rotor under certain circumstance. A "horizontal particle" was proved to be more suitable for measurement compared to a "vertical particle".

The analysis and simulation of an optically levitated rotating particle are essential in torque measurement. An optically levitated particle experiences none of the machinery's friction, presenting great prospects for its being treated as a high-speed rotor. Our work gave a detailed description of the motion process of a particle, laying the foundation for the further motion analysis.

\section{Acknowledgment}

Our research is supported by Major Scientific Research Project of 378 Zhejiang Lab (Grant No. 2019MB0AD01) and National Program for Special Support of Top-Notch Young Professionals, Fundamental Research Funds for the Central Universities 380 (Grant Nos. 2016XZZX004-01 and 2018 XZZX001-08)

Open Access This article is distributed under the terms of the Creative Commons Attribution 4.0 International License (http://creativecommons.org/licenses/by/4.0/), which permits unrestricted use, distribution, and reproduction in any medium, provided you give appropriate credit to the original author(s) and the source, provide a link to the Creative Commons license, and indicate if changes were made.

\section{References}

[1] A. Ashkin and J. M. Dziedzic, "Feedback stabilization of optically levitated particles," Applied Physics Letters, 1977, 30(4): 202-204.

[2] A. La Porta and M. D. Wang, "Optical torque wrench: angular trapping, rotation, and torque detection of quartz microparticles," Physical Review Letters, 2004, 92(19): 190801.

[3] M. E. J. Friese, T. A. Nieminen, N. R. Heckenberg, and H. Rubinsztein-Dunlop, "Optical torque controlled by elliptical polarization," Optics Letters, 1998, 23(1): 1-3.

[4] S. Kuhn, B. A. Stickler, A. Kosloff, F. Patolsky, K. Hornberger, M. Arndt, et al., "Optically driven ultra-stable nanomechanical rotor," Nature Communications, 2017, 8(1): 1-5. 
[5] Y. Arita, J. M. Richards, M. Mazilu, G. C. Spalding, S. E. Skelton Spesyvtseva, D. Craig, et al., "Rotational dynamics and heating of trapped nanovaterite particles," ACS Nano, 2016, 10(12): 11505-11510.

[6] E. Higurashi, R. Sawada, and T. Ito, "Optically induced angular alignment of trapped birefringent micro-objects by linearly polarized light," Physical Review E, 1999, 59(3): 3676-3681.

[7] F. Monteiro, S. Ghosh, E. C. van Assendelft, and D. C. Moore, "Optical rotation of levitated spheres in high vacuum," Physical Review A, 2018, 97(5): 051802 .

[8] R. Reimann, M. Doderer, E. Hebestreit, R. Diehl, M. Frimmer, D. Windey, et al., "GHz rotation of an optically trapped nanoparticle in vacuum," Physical Review Letters, 2018, 121(3): 033602.

[9] J. Ahn, Z. Xu, J. Bang, Y. H. Deng, T. M. Hoang, Q. Han, et al., "Optically levitated nanodumbbell torsion balance and $\mathrm{GHz}$ nanomechanical rotor," Physical Review Letters, 2018, 121(3): 033603.

[10] P. Polimeno, A. Magazzù, M. AntoniaIatì, F. Patti, R. Saija, C. D. E. Boschi, et al., "Optical tweezers and their applications," Journal of Quantitative Spectroscopy and Radiative Transfer, 2018, 218: $131-150$.

[11] A. V. Arzola, P. Jákl, L. Chvátal, and P. Zemánek, "Rotation, oscillation and hydrodynamic synchronization of optically trapped oblate spheroidal microparticles," Optics Express, 2014, 22(13): 16207-16221.

[12] K. Hein, T. Hucke, M. Stintz, and S. Ripperger, "Analysis of adhesion forces between particles and wall based on the vibration method," Particle \& Particle Systems Characterization, 2002, 19(4): 269-276.

[13] J. S. Bennett, L. J. Gibson, R. M. Kelly, E. Brousse, B. Baudisch, D. Preece, et al., "Spatially-resolved rotational microrheology with an optically-trapped sphere," Scientific Reports, 2013, 3(1): 1-5.

[14] A. Raghu and S. Ananthamurthy "Optical tweezer for micro and nano scale rheology of biomaterials," Indian Journal of Physics, 2010, 84(8): 1051-1061.

[15] T. A. Nieminen, A. I. Bishop, N. R. Heckenberg, and H. Rubinsztein-Dunlop, "Polarimetric measurement of optical torque," in 7th Conference on Electromagnetic and Light Scattering by Nonspherical Particles: Theory, Measurements, and Applications, Bremen, 2011, pp. 267-270.

[16] W. Li, N. Li, Y. Shen, Z. Fu, H. Su, and H. Hu, "Dynamic analysis and rotation experiment of an optical-trapped microsphere in air," Applied Optics, 2018, 57(4): 823-828.

[17] A. T. M. A. Rahman and P. F. Barker, "Laser refrigeration, alignment and rotation of levitated $\mathrm{Yb}^{3+}$ : YLF nanocrystals," Nature Photonics, 2017, 11(10): 634-638.

[18] J. W. Liaw, W. J. Lo, W. C. Lin, and M. K. Kuo, "Theoretical study of optical torques for aligning $\mathrm{Ag}$ nanorods and nanowires," Journal of Quantitative Spectroscopy and Radiative Transfer, 2015, 162: 133-142.

[19] R. Ali, F. A. Pinheiro, R. S. Dutra, F. S. S. Rosa, and P. A. M. Neto, "Enantioselective manipulation of single chiral nanoparticles using optical tweezers," Nanoscale, 2020, 12(8): 5031-5037.

[20] T. M. Hoang, Y. Ma, J. Ahn, J. Bang, F. Robicheaux, Z. Q. Yin, et al., "Torsional optomechanics of a levitated nonspherical nanoparticle," Physical Review Letters, 2016, 117(12): 123604.

[21] S. Kuhn, A. Kosloff, B. A. Stickler, F. Patolsky, K. Hornberger, M. Arndt, et al., "Full rotational control of levitated silicon nanorods," Optica, 2017, 4(3): 356-360.

[22] M. Rashid, M. Toroš, A. Setter, and H. Ulbricht, "Precession motion in levitated optomechanics," Physical Review Letters, 2018, 121(25): 253601.

[23] J. Bang, T. Seberson, P. Ju, J. Ahn, Z. Xu, X. Gao, et al., "Five-dimensional cooling and nonlinear dynamics of an optically levitated nanodumbbell," Physical Review Research, 2020, 2(4): 043054.

[24] A. A. M. Bui, A. B. Stilgoe, I. C. D. Lenton, L. J. Gibson, A. V. Kashchuk, S. Zhang, et al., "Theory and practice of simulation of optical tweezers," Journal of Quantitative Spectroscopy \& Radiative Transfer, 2017, 195: 66-75.

[25] M. E. J. Friese, T. A. Nieminen, N. R. Heckenberg, and H. Rubinsztein-Dunlop, "Optical alignment and spinning of laser-trapped microscopic particles," Nature, 1998, 394(6691): 348-350.

[26] T. A. Nieminen, H. Rubinsztein-Dunlop, and N. R. Heckenberg, "Calculation and optical measurement of laser trapping forces on non-spherical particles," Journal of Quantitative Spectroscopy \& Radiative Transfer, 2001, 70(4-6): 627-637.

[27] F. van der Laan, R. Reimann, A. Militaru, F. Tebbenjohanns, D. Windey, M. Frimmer, et al., "Optically levitated rotor at its thermal limit of frequency stability," Physical Review A, 2020, 102(1): 013505 .

[28] M. Ivanov and D. Hanstorp, "Controlled spin of a nonbirefringent droplet trapped in an optical vortex beam," Optics Communications, 2018, 427: 152-157.

[29] A. I. Bishop, T. A. Nieminen, N. R. Heckenberg, and H. Rubinsztein-Dunlop, "Optical microrheology using rotating laser-trapped particles," Physical Review Letters, 2004, 92(19): 198104. 